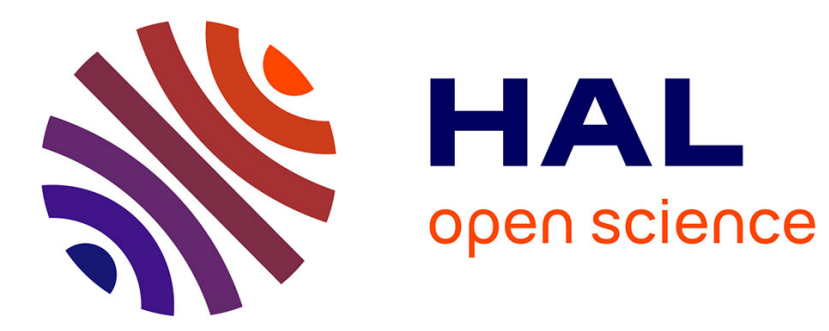

\title{
EXCITATION OF "M1 TRANSITIONS" IN INELASTIC PROTON SCATTERING
}

\author{
C. Djalali
}

\section{To cite this version:}

C. Djalali. EXCITATION OF "M1 TRANSITIONS" IN INELASTIC PROTON SCATTERING. International Symposium on Highly Excited States and Nuclear Structure Hesans 83, 1983, Orsay, France. pp.C4-375-C4-387, 10.1051/jphyscol:1984429 . jpa-00224095

\section{HAL Id: jpa-00224095 https://hal.science/jpa-00224095}

Submitted on 1 Jan 1984

HAL is a multi-disciplinary open access archive for the deposit and dissemination of scientific research documents, whether they are published or not. The documents may come from teaching and research institutions in France or abroad, or from public or private research centers.
L'archive ouverte pluridisciplinaire HAL, est destinée au dépôt et à la diffusion de documents scientifiques de niveau recherche, publiés ou non, émanant des établissements d'enseignement et de recherche français ou étrangers, des laboratoires publics ou privés. 


\title{
EXCITATION OF "MI TRANSITIONS" IN INELASTIC PROTON SCATTERING
}

\author{
C. Djalali \\ Institut de Physique Nucléaire, B.P. nº, 91406 Orsay, France
}

Résumé : La diffusion inélastique des protons de $201 \mathrm{MeV}$, aux petits angles est très sélective pour exciter les transitions magnétiques $\Delta L=0$. Ceci est démontré par comparaison avec les mesures faites en $\left(p, p^{\prime}\right)$ à $65 \mathrm{MeV}$ et en $\left(e, e^{\prime}\right)$. Les résultats obtenus sur les isotopes du calcium, les isotones $N=28$, ${ }^{8} \mathrm{Sr}$ et ${ }^{90} \mathrm{Zr}$ sont présentés. On discute les différences qui apparaissent entre Tes distributions relatives de 1 a force $M 1$ obtenues en $\left(p, p^{\prime}\right)$ et en $\left(e, e^{\prime}\right)$. Des rësultats préliminaires sur le ${ }^{208} \mathrm{~Pb}$ sont donnés; le niveau $1^{+}$"isoscalaire" à 5,846 MeV est excité.

\begin{abstract}
The high selectivity of $201 \mathrm{MeV}$ inelastic proton scattering at forward angles for exciting $\Delta L=0$ spin flip transitions is outlined by comparison with $\left(e, e^{\prime}\right)$ and $65 \operatorname{MeV}\left(p, p^{\prime}\right)$ measurements. A summary of the results obtained on the calcium isotopes, the $N=28$ isotones, ${ }^{88} \mathrm{Sr}$ and ${ }^{90} \mathrm{Zr}$ is presented. The differences in the relative M1 strength distributions between the $\left(p, p^{\prime}\right)$ and the $\left(e, e^{\prime}\right)$ results are discussed. Preliminary results on ${ }^{208} \mathrm{~Pb}$ are given ; the "isoscalar" $1^{+}$state at $5.846 \mathrm{MeV}$ is excited.
\end{abstract}

\section{I - INTRODUCTION}

With the availability of new intermediate energy proton beams and electron beams with improved energy resolution, the study of spin degrees of freedom in nuclei has become one of the most active areas of research in nuclear physics. High resolution backward electron scattering (1), charge exchange $(p, n)$ reactions (2), nuclear resonance fluorescence (3) and proton inelastic scattering both at low (4) and intermediate energies (5) have allowed us to acquire a detailed knowledge on spin excitation strength and on the degree to which spins are paired in the nuclear ground state. One of the most interesting features which has emerged from the study of either the Gamow-Teller (G.T.) or the M1 transitions is that a significant fraction of the expected strength is missing in medium and heavy nuclei. Equally as exciting as these experimental discoveries are the consequent theoretical developments which seem to open new perspectives beyond the scope of conventional nuclear $(p-h)$ excitation and the inclusion of the $\Delta$ (1232) resonance degrees of freedom in low energy nuclear processes (6). However recent elaborate calculations (7) point out that in order to explain the missing strength the second order core polarisation produced by short range tensor forces is found to be as important as, and even in some cases more important, than the isobar currents.

Until recently, there had been a long standing problem to as why both $\left(e, e^{\prime}\right)$ and low energy $\left(p, p^{\prime}\right)$ experiments do not find substantial M1 strength in nuclei with $A>60$. This situation became even more puzzling when intermediate energy $(p, n)$ reactions found large amount of (G.T) strength in all nuclei till ${ }^{208} \mathrm{~Pb}$. The $\left(p, p^{\prime}\right)$ experiments (5) (8) performed at the Orsay Synchrocyclotron at $201 \mathrm{MeV}$ with a good energy resolution ( 50 to $60 \mathrm{keV}$ ) and the possibility of doing measurements at angles as small as $2^{\circ}$, had resolved this problem by exciting large MI resonances in medium heavy nuclei.

A more recent emphasis has arised from the agreements and disagreements that are observed in the relative M1 strength distributions measured by different probes. 
By detailed comparisons, one can expect to get a better understanding of the spin degrees of freedom in nuclei. In this paper, $\left(p, p^{\prime}\right)$ results obtained at the orsay Synchrocyclotron at $201 \mathrm{MeV}$ are presented. Comparisonswith the $\left(e, e^{\prime}\right),\left(\vec{\gamma}, \gamma^{\prime}\right)$ and the $65 \mathrm{MeV}\left(p, p^{\prime}\right)$ results are made.

\section{II - REACTION MECHANISM}

In the simple Shell Model, an M1 transition is described as a single particle-hole $(p-h)$ transition between unsaturated spin orbit partner levels $i . e\left(j_{>}=l+1 / 2 \rightarrow j_{<}=\right.$ $\ell-1 / 2)$. M1 transitions involve a spin transfer $\Delta S$ of one and an orbital momentum transfer $\Delta L$ either of zero or two, resulting in a total spin transfer $\Delta J$ of one without change in the parity. (In even-even nuclei, Mltransitions lead to $I^{+}$states). Furthermore if an isospin transfer $\Delta T$ of one or zero $\left(\Delta T_{2}=0\right)$ is involved, the transitions are called respectively isovector or isoscalar. The expression "MI transition" properly refers only to a transition induced by the electromagnetic $M 1$ operator (see II, 4), however let us, for convenience, extend it to all transitions involving the quantum number mentioned above. In inelastic proton scattering, the effective interaction between the incoming proton and the target nucleon can be parametrized in the following way (9):

$$
\begin{aligned}
V_{\text {eff }}=V_{\text {couT }} & +V_{0}+V_{\tau}\left(\vec{\tau}_{1} \cdot \vec{\tau}_{2}\right)+V_{\sigma}\left(\vec{\sigma}_{1} \cdot \vec{\sigma}_{2}\right)+V_{\sigma \tau}\left(\vec{\sigma}_{1} \cdot \vec{\sigma}_{2}\right)\left(\vec{\tau}_{1} \cdot \vec{\tau}_{2}\right)+\left[V_{L S}+V_{L S}\left(\vec{\tau}_{1} \cdot \vec{\tau}_{2}\right)\right] \overrightarrow{L S}_{L} \\
& +\left[V_{T}+V_{T \tau}\left(\vec{\tau}_{1} \cdot \vec{\tau}_{2}\right)\right] S_{12}
\end{aligned}
$$

where $S_{12}$ is the tensor term and $V_{c o u l}$ the coulomb term. In $(p, n)$ reactions only the isovector terms are present, in $(p, p)$ the isoscalar terms also contribute.

1) Energy dependence

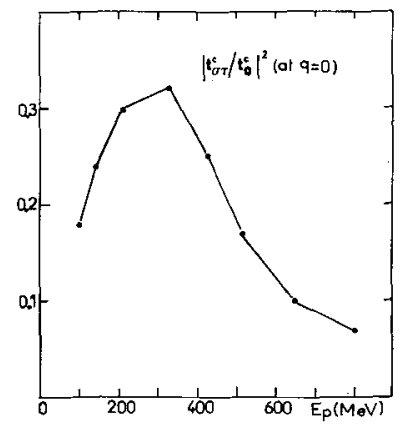

Fig. 1 : The energy dependence of $\left|t_{\sigma \tau}\right|$ and $\left|t_{0}\right|$ is taken from ref. 10 .
Taking the energy dependence of the nucleon-nucleon interaction given by Love and Franey (10), we have plotted in Fig. 1, as a function of the bombarding energy and for the momentum transfer $q=0$, the square of the ratio between t-matrix elements in the $\sigma \tau$ and in the central spin independent channels.

The spin-isospin term $t_{\sigma \tau}$ decreases very slowly as one goes from $100 \mathrm{MeV}$ to $800 \mathrm{MeV}$, while the spin independent central part $t$ which is the dominant term at all energies, has a pronounced minimum for energies between 200 and $400 \mathrm{MeV}$. This is the reason why around $200 \mathrm{MeV}$, spin-flip transitions are enhanced relatively to natural parity transitions. For proton bombarding energies between 200 and $400 \mathrm{MeV}$ and at very small momentum transfer, the $M I$ transition operator reduces essentially to the $V_{O T}$ term plus a central and a tensor isoscalar spin flip term which are not completely negligible. Nevertheless the inclusion of these terms makes only about a $20 \%$ difference to the calculated crosssection. It is then a reasonably good approximation to say that the $\left(p, p^{\prime}\right)$ reaction for exciting $(\Delta L=0, \Delta S=1)$ states is dominated by the Vot term.

\section{2) Angular distributions}

Around $200 \mathrm{MeV}$, the angular distributions are very characteristic of the orbital momentum transfer. In fia. 2 are plotted DWBA-angular distributions calculated with a surface peaked macroscopic form factor for several $\Delta \mathrm{L}$ transfers. Only the $\Delta \mathrm{L}=0$ transfer has a forward peaked angular distribution and is therefore enhanced at small angles, relatively to higher $\Delta \mathrm{L}$ transfers. 
At lower bombarding energies (around $50 \mathrm{MeV}$ ), the angular distributions are much less characteristic of the $\Delta L$-transfer.

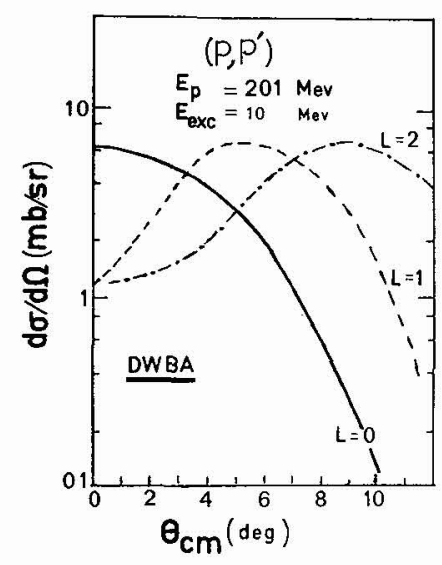

Fig.2 : Macroscopic DWBA angular distributions.
At intermediate energies, $M 1$ transitions have a very forward peaked angular distribution. This is seen clearly in Fig. 3 where spectra from ${ }^{51} V\left(p, p^{\prime}\right)$ (8) are shown at laboratory angles of $3^{\circ}$ and $8^{\circ}$. The large M1 resonance, the analogue of which has been recentiy observed in a $(p, n)$ reaction (11) has practically disappeared at $8^{\circ}$. The measured angular distribution is fitted rather well by a macroscopic DWBA $(\Delta L=0)$ calculation. We see how crucial it is to be able to do measurementsat very forward angles otherwise even if we have a favorable bombarding proton energy we could easily miss the M1 transition. Let me stress that measurements of inelastic scattering cross sections at forward angles are absolutely not straightforward and are in fact experimentally very challenging.

In order to illustrate the high selectively for M1 transitions at incident energies around $200 \mathrm{MeV}$, we have compared in Fig. 4 three spectra on $50 \mathrm{Ti}$ respectively obtained in a $65 \mathrm{MeV}\left(p, p^{\prime}\right)$ experiment (12), a $201 \mathrm{MeV}\left(p, p^{\prime}\right)$ experiment (13) and a $30 \mathrm{MeV}$ $\left(e, e^{i}\right)$ experiment $(1)$.

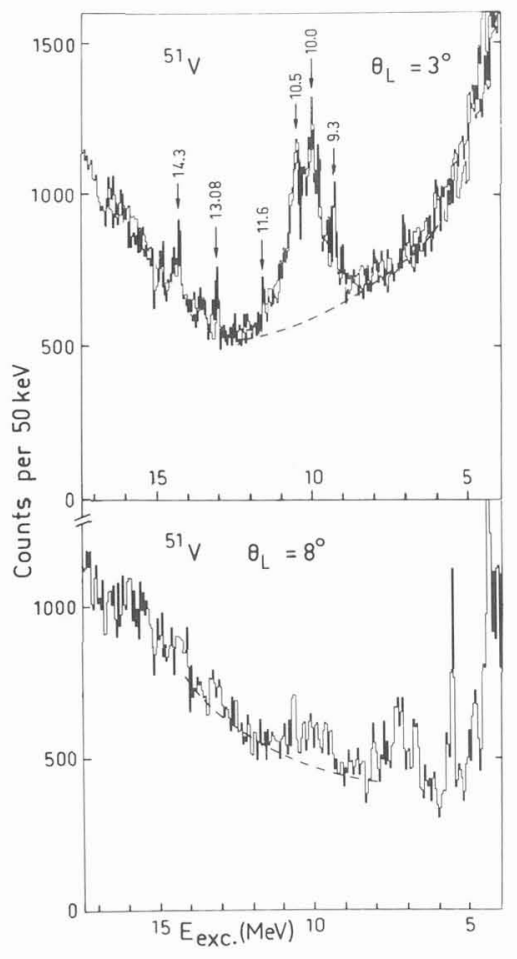

Fig.3: ${ }^{51} \mathrm{~V}\left(\mathrm{p}, \mathrm{p}^{\prime}\right)$ spectra at $201 \mathrm{MeV}$.

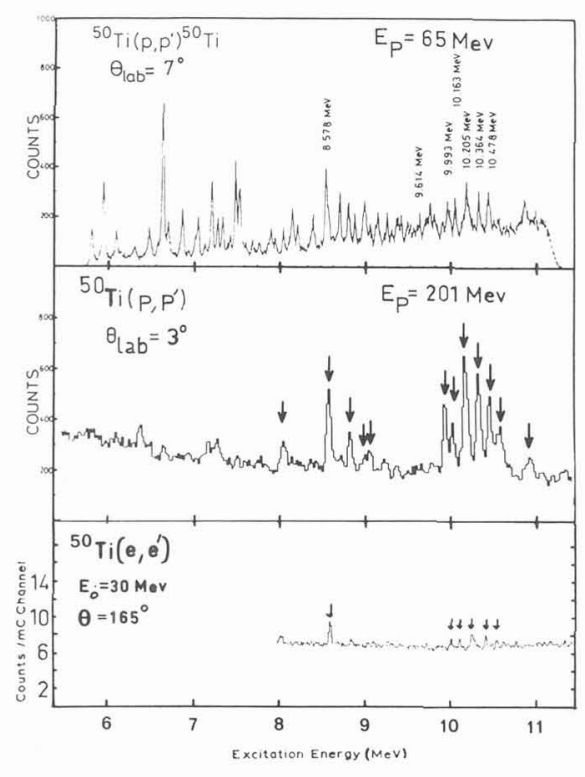

Fig. 4 : Comparison of the excitation of $1^{+}$states (pointed by arrows) in different reactions : ( $\left.p, p^{\prime}\right)$ at $65 \operatorname{MeV}(12),\left(p, p^{\prime}\right)$ at $201 \mathrm{MeV}(13)$ and $\left(e, e^{\prime}\right)$ at $30 \mathrm{MeV}(1)$. 
At $65 \mathrm{MeV}$, the central spin independent part of the interaction (i.e. $V_{0}$ ) is by far the dominant term, leading to a lack of selectivity for spin-flip transitions. Consequently serious difficulties in studying $1^{+}$states arise from the strong excitation of a large number of natural parity states, added to the fact that the angular distributions are not very characteristic. However, the very good energy resolution (10 to $15 \mathrm{keV}$ ) allows the separation of the strongest known $1^{+}$states. At $201 \mathrm{MeV}$, the favorable incident energy combined with the fact that $\Delta L=0$ transfers are enhanced at forward angles leads to the spectrum shown in Fig. 4 where almost all the structures observed are $1^{+}$states. In this case the selectivity for exciting $1^{+}$ states is comparable to that of the backward inelastic electron scattering (given in the lower part of Fig. 4). In addition the ratio "peak to continuum" in the ( $\left.p, p^{\prime}\right)$ experiment is almost ten times larger than the ratio observed in the $\left(e, e^{\prime}\right)$ experiment.

\section{3) $3^{\pi}$ ambiguities}

A $\Delta L=0$ angular distribution only implies that the spin parity of the excited state (in even-even nuclei) is $0^{+}$or $1^{+}$. In the cases, where the M1 nature of the observed transition is not unambiauously determined by other highly selective experiments like $\left(\vec{\gamma}, \gamma^{\prime}\right)$ or $\left(e, e^{\prime}\right)$, let me briefly summarise the arguments that are set forth to rule out the $0^{+}$nature :

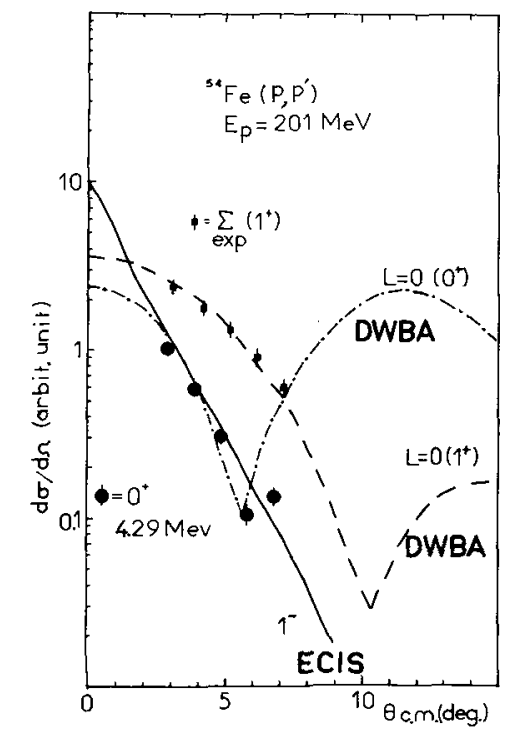

Fig. 5 : The measured crosssection for the $1^{+}$resonance and a $0^{+}$state are compared to different theoretical calculations (see text). -i) When available, the most conclusive is of course the non observation of such a transition in an $\left(\alpha, \alpha^{\prime}\right)$ experiment.

-ii) If the state were a $0^{+}$, it should have been excited at lower incident energies as the central spin independent part of the interaction is much stronger than at $200 \mathrm{MeV}$.

-iii) In light nuclei (till $A=60$ ) where known $0^{+}$states are observed, they generally have a much steeper angular distribution than that of $1^{+}$states. This is the case in ${ }^{54} \mathrm{Fe}$ (Fig. 5) where in order to reproduce the angular distribution of the $0^{+}$state, we have to use a volume transition density in the DUBA calculation.

Life would have been easier if we were to deal only with the nuclear interaction, but it has been shown (14) that $201 \mathrm{MeV}$ proton inelastic scattering strongly excites Isovector electric dipole transitions through the coulomb interaction. The angular distributions for such trans itions àre also very forward peaked. In light nuclei, the ( $E 1, \Delta T=1)$ coulomb excited angular distribution is much steeper than the $1^{+}$angular distribution (see Fig. 5). However as we move to heavier nuclei both angular distributions become almost identical at forward angles.

The best way to handle the Coulomb excited $1^{-}$states, is to use, when available the results of the very nice $\left(\vec{\gamma}, \gamma^{\prime}\right)$ measurements (3). From the excitation energy and the de-excitation width $\left(\Gamma_{0}\right)$ of the $1^{-}$state, reported in the $\left(\vec{\gamma}, \gamma^{\prime}\right)$ experiment, the transition probability $B(E 1)$ is deduced; using the code ECIS 79 (15) one is able to predict very accurately the $\left(p, p^{\prime}\right)$ cross sections for this $1^{-}$state. We shall see that in light nuclei, the strongest $1^{-}$states reported in $\left(\vec{\gamma}, \gamma^{\prime}\right)$ reactions, have $\left(p, p^{\prime}\right)$ cross-sections at the limit of detectability. In heavier nuclei like ${ }^{208} \mathrm{pb}$, the situation is quite different and at forward angles, almost all the dominant peaks are due to $(E 1, \Delta T=1)$ transitions. 


\section{4) Comparison with $\left(e, e^{\prime}\right)$ and $\left(\vec{\gamma}, \gamma^{\prime}\right)$ results}

For electromagnetic interactions, the MI operator includes both a spin and an orbital term :

$$
\mathcal{O}(M 1)=\sum_{k=1}^{A} g_{S} \vec{s}(k)+g_{\ell} \vec{\ell}(k)
$$

whereas in ( $\left.p, p^{\prime}\right)$ scattering at forward angles and at $200 \mathrm{MeV}$, only the spin term $\sigma \tau$ contributes. Only in the cases of pure neutron excitations, the orbital term disappears in the electromagnetic M1 operator, then there should be a close analogy between the relative strengths observed in $\left(e, e^{\prime}\right)$ or $\left(\vec{\gamma}, \gamma^{\prime}\right)$ and $\left(p, p^{\prime}\right)$. In general both proton and neutron excitations contribute and as the relative weight of the various contributions to the M1 transition vary from state to state, one should expect to observe differences in the relative strength distributions. Such differences can, in principle, be used to extract information on the nature of particular transitions.

\section{5) The observed strength}

Concerning the observed $1^{+}$strength in $\left(p, p^{\prime}\right)$ experiments, let us review the main sources of uncertainties.

Generally the observed Mi excitations appear on the top of a continuum, the shape and the height of which must be estimated in order to extract the $1^{+}$cross sections. Continuum calculations like those done by osterfeld (16) for $(p, n)$ reactions, unfortunately are presently not available for $\left(p, p^{\prime}\right)$ reactions. Thus the usual procedure of drawing an empirical smooth continuum is adopted. This kind of background subtraction can introduce in the case of broad resonances experimental uncertainties as large as $20 \%$. We can also miss very fragmented strength below our limit of detectability. In $(p, n)$ reactions, the measured G.T. Strength can be compared to a model independent Sum Rule (17). This is not the case in $\left(p, p^{\prime}\right)$ reactions where the predicted strength is strongly model dependent. The analysis is done within the Distorded Wave Impulse Approximation (D.W.I.A) using the following ingredients : i) an optical potential; ii) a nucleon-nucleon interaction or phase shifts, $i$ i) a model wave function. It is in the latter ingredient that the strong mode 1 dependence enters. By comparing the measured cross sections to the calculated ones, we deduce how much strength is observed.

\section{III - EXPERIMENTAL RESULTS}

1) The Calcium isotopes.

Following the pure independent particle shell model, no M1 transitions should occur in ${ }^{40} \mathrm{Ca}$ because the shells of both spin orbit partners are filled. When we proceed from ${ }^{42} \mathrm{Ca}$ to ${ }^{48} \mathrm{Ca}$, as we fill the $\mathrm{f}_{7 / 2}$ neutron shel1, stronger and stronger $\left[v\left(f_{5 / 2}, f_{7 / 2}^{-1}\right)\right]^{1+}$ transitions are expected, with a maximum in ${ }^{48} \mathrm{Ca}$ where the $f_{7 / 2}$ neutron she 11 is closed. The very nice $\left(e, e^{\prime}\right)$ data taken at Darmstatd (18) on the calcium isotopes have prompted $\left(p, p^{\prime}\right)$ measurements on the same nuclei (19). The spectra obtained at $201 \mathrm{MeV}$ are shown in Fig. 6.

${ }^{48} \mathrm{C}$ a : The high selectivity of the $\left(p, p^{\prime}\right)$ reaction at $201 \mathrm{MeV}$ is seen in the lower part of Fig. 6 , where at $3^{\circ}$ apart from the single strong $1^{+}$state at $10.22 \mathrm{MeV}$, no other structure is observed. The strong $1^{+}$state was first observed in backward angle electron scattering (18). In the present experiment the ratio peak to continuum at forward angles is about eight times greater than the same ratio observed in the $\left(e, e^{\prime}\right)$ experiment. In addition to the $10.22 \mathrm{MeV}$ state, weak $1^{+}$states have been reported (18). In a $\left(p, p^{\prime}\right)$ reaction at $65 \mathrm{MeV}(4), a 1^{+}$state is reported at $9.0 \mathrm{MeV}$ with about $10 \%$ of the strength of the $10.22 \mathrm{MeV}$ state. This state is not observed in the present experiment done with very good statistics. Furthermore, no other definite $1^{+}$state has been identified, the possible additionnal $1^{+}$strength, spread 


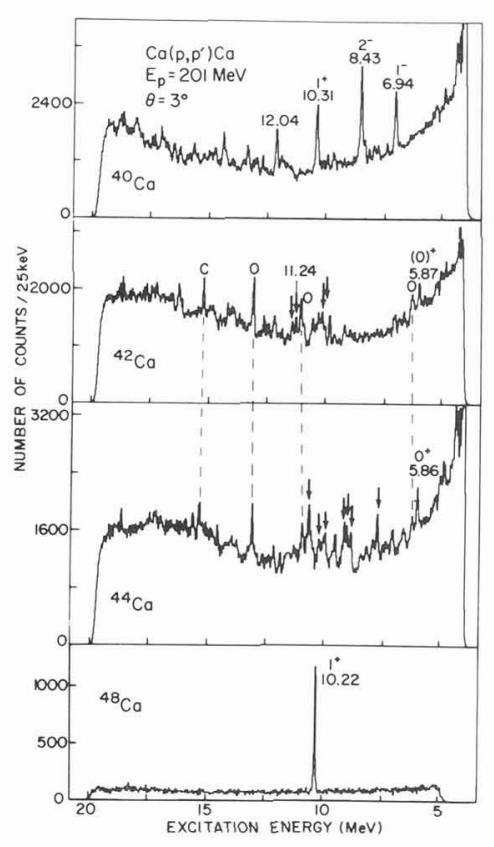

Fig. $6:\left(p, p^{\prime}\right)$ spectra on the Ca isotopes.

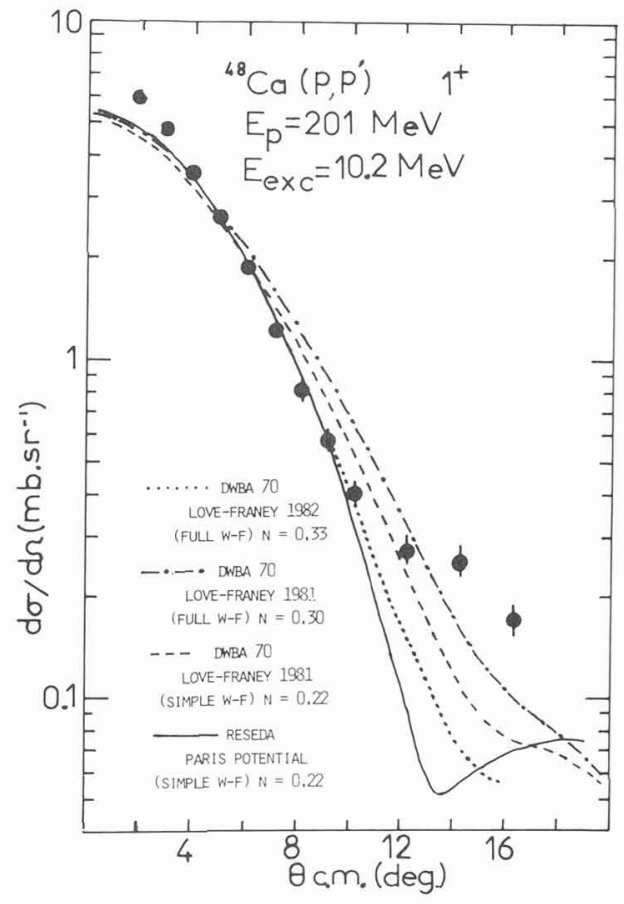

Fig. 7 : Angular distributions for $\left(p, p^{\prime}\right)$ to the $10.22 \mathrm{MeV}$ state of ${ }^{48} \mathrm{Ca}$. The curves are calculations described in the text.

in small peaks between 7 and $13 \mathrm{MeV}$ of excitation energy, corresponds at most to $35 \%$ of the strength observed in the $10.22 \mathrm{MeV}$ state.

${ }^{48} \mathrm{Ca}$ is the ideal case to study the missing M1 strength because : i) as we have a strong sharp $1^{+}$state, there is little ambiguity in the background subtraction and the experimental cross sections can be obtained quite accurately, ii) ${ }^{48} \mathrm{Ca}$ is believed to have a simple structure and realistic wave functions are available (21).

In fig. 7 , the measured cross sections for the $1^{+}$state at $10.22 \mathrm{MeV}$ is compared to several microscopic distorded wave calculations carried out using both DWBA70 (G) and RESEDA (22) codes. In the DWBA 70 calculation, the full Love and Franey parametrization of the nucleon-nucleon interaction at $210 \mathrm{MeV}$ (10) was used with an exact treatment of the exchange terms. The RESEDA calculations were done with the nucleonnucleon phase shifts from the PARIS potential (23). Using the simple wave function $\left[v\left(f_{5 / 2}, f_{7 / 2}^{-1}\right)\right]^{+1}$, the RESEDA calculation fits the data better than the DWBA 70 calculation, however, in order to match the data both calculations, have been normalized by $N=0.22$. If we take a full $f-p$ shell wave function (21), the normalization factor becomes 0.30 . More recently a further DWBA 70 calculation was made with a new set of unpublished parameters from Love and Franey (24), it gives a value of $\mathrm{N}$ equal to 0.33 . We see in Fig. 7 , that all the calculations fail to reproduce the data beyond $10^{\circ}$. One possible explanation for this discrepancy is the presence of levels of higher multipolarity very close to the $10.22 \mathrm{MeV}$ state (20) (25) which have not been resolved at large angies, in this experiment. Concerning the observed 
strength, if we restrict ourselves to the strong state at $10.22 \mathrm{MeV}$, on $1 \mathrm{y} 30$ to $33 \%$ of the predicted strength is observed. If we add the possible $1^{+}$states, spread between 7 and $13 \mathrm{MeV}$ of excitation energy, the total measured $1^{+}$strength sums up atmost to $40 \%$ of the predicted one. For a more complete discussion about the missing $1^{+}$strength in ${ }^{48} \mathrm{Ca}$, see reference 19$)$.

${ }^{40} \mathrm{Ca}$ : In agreement with $\left(e, e^{1}\right), a 1^{+}$state at $10.32 \mathrm{MeV}$ is clearly excited at forward angles and has a $\Delta \mathrm{L}=0$ angular distribution. This state is explained in terms of ground state correlations. Another state with the same angular distribution is excited at $12.04 \mathrm{MeV}$. The $1^{+}$assignment is inconsistent with the non observation of this level in the (e, $\left.e^{\prime}\right)$ reaction. Furthermore, in a recent $\left(\alpha, \alpha^{\prime}\right)$ reaction (26), no strong $0^{+}$state is excited at the same energy. The nature of this $12.04 \mathrm{MeV}$ state obviously requires further clarification.

${ }^{42} \mathrm{Ca}-{ }^{44} \mathrm{Ca}$ : In these two isotopes, contrary to what one might have expected from the simple independent particle shell model, no strong $1^{+}$state is observed. We see in Fig. 6 that apart from the contaminant oxygen and carbon peaks, the spectra do not exhibit any strong state. The sma 11 peaks which are possible $1^{+}$states are denoted by arrows.

In ${ }^{42} \mathrm{Ca}$, the $\left(e, e^{\prime}\right)$ experiment reports on $7 y$ one strong $1^{+}$state at 11.235 MeV having a strength of about $1 / 7$ of that of the $1^{+}$state in ${ }^{48} \mathrm{Ca}$. In the present $\left(p, p^{\prime}\right)$ experiment, such a state is not observed, the upper limit on the cross sections for a state at $11.24 \mathrm{MeV}$ would be of about $1 / 30$ of the strength of the $1^{+}$state in ${ }^{48} \mathrm{Ca}$. This result is in agreement with the ${ }^{42} \mathrm{Ca}(p, n)$ experiment (27) where no analogue of the $11.24 \mathrm{MeV}$ state is observed. Furthermore, in the present ( $p, p^{\prime}$ ) experiment, other weak $1^{+}$states are excited respectively at $10.08,10.24$ and $11.40 \mathrm{MeV}$. The two close lying peaks at 10.08 and $10.24 \mathrm{MeV}$ may be the parent states of the $1^{+}$level seen in the ${ }^{42} \mathrm{Ca}(p, n)$ reaction $(27)$. The known $0^{+}$state at $5.87 \mathrm{MeV}$ has an angular distribution much steeper than that of the $1^{+}$states. In ${ }^{44} \mathrm{Ca}$, the $1^{+}$strength is very fragmented. Very weak $1^{+}$states are observed at $7.60,8.73,9.01,9.42,10.01$, 10.25 and $10.58 \mathrm{MeV}$. This 7 ist is preliminary and not exhaustive (28). A 71 these states have the same angular distribution as the $1^{+}$state in ${ }^{48} \mathrm{Ca}$. Again, as in ${ }^{42} \mathrm{Ca}$, the known. $0^{+}$state at $5.86 \mathrm{MeV}$ has a much steeper angular distribution. These known $0^{+}$states illustrate the discussion given in section $\left.\left.I I, 2\right), \quad i j i\right)$.

\section{2) The $N=28$ Isotones}

The $N=28$ nuclei have been extensively studied by $\left(e, e^{\prime}\right)(1),\left(\vec{\gamma}, \gamma^{\prime}\right)(3)$ and low energy $\left(p, p^{\prime}\right)(4)$ reactions. In addition shell model calculations by Metsch and Knüpfer (29) are available for all these isotones. It is interesting to observe the effect of adding extra protons to the $f_{7}$ shell while keeping the neutron number constant at 28 . Sfectra from ${ }^{50} \mathrm{Ti},{ }^{52} \mathrm{Cr}$ and ${ }^{54} \mathrm{Fe}$ taken at $3^{\circ}$ in the $201 \mathrm{MeV}\left(\mathrm{p}, \mathrm{p}^{\prime}\right)$ reaction are shown in Fig. 8 , together with a ${ }^{48} \mathrm{Ca}$ spectrum for comparison. For all the targets, nearly ail the states observed at small angles between 8 and $15 \mathrm{MeV}$ excitation energy have a forward peaked angular distribution and have been identified as $1^{+}$states. This illustrates once more the high selectivity of $200 \mathrm{MeV}$ proton scattering for spin-flip transitions.

As one adds protons in the $f_{7 / 2}$ shel1 in moving to ${ }^{54} \mathrm{Fe}$, the $1^{+}$strength which appears in ${ }^{4: 8} \mathrm{Ca}$ in a single state (mainly due to $\nu\left(f_{5 / 2}, f_{7 / 2}^{-1}\right.$ ) excitation) splits up into many states. In ${ }^{50} \mathrm{Tj}$, two clusters of states are clearly visible in Fig. 8. Near $10 \mathrm{MeV}$, there are at least seven states; the more important ones have aiready been reported in the $\left(p, p^{\prime}\right)$ experiment at $65 \mathrm{MeV}(4)$ and in the $\left(e, e^{\prime}\right)$ experiment (1). The centroid energy of these states as well as their sumned cross section is very close to those of the ${ }^{48} \mathrm{Ca} 1^{+}$state. Thus, all the states in this cluster can be considered to arise mainly from the splitting of the $\nu\left(f_{5 / 2}, f_{7 / 2}^{-1}\right)$ configuration. Around $8.5 \mathrm{MeV}$, there are at least four $1^{+}$states. Only the most strongly excited one at $8.56 \mathrm{MeV}$ has been reported in other experiments. This cluster of states is predicted by the model to arise mainly from proton excitation of the type 
$\pi\left(f_{5 / 2}, f_{7 / 2}^{-1}\right)$

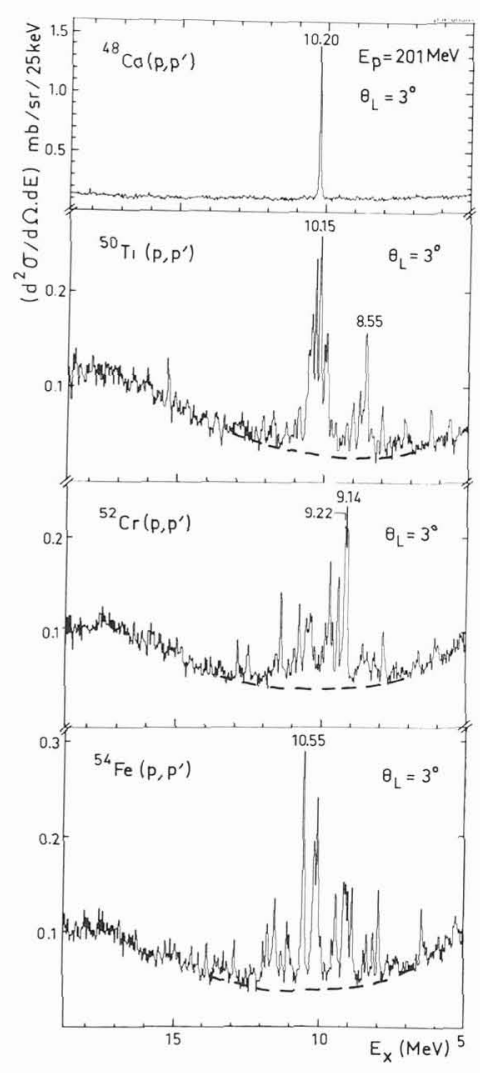

Fig. $8:\left(p, p^{\prime}\right)$ spectra on the $\mathrm{N}=28$ isotones.
In ${ }^{52} \mathrm{Cr}$ and ${ }^{54} \mathrm{Fe}$, the splitting of the strength is more pronounced than in ${ }^{50} \mathrm{~T} \hat{\mathrm{j}}$, though it is no more possible to groupe the states into proton and neutron configurations. The energies of the ma in peaks are in good aareement with those observed in the $\left(e, e^{\prime}\right)$ experiment. The peak to continuum ratio is 10 to 15 times larger in the $\left(p, p^{\prime}\right)$ experiment than in the (e,e') experiment. ${ }^{52} \mathrm{Cr}$ is a good case to iliustrate that $\left(i^{-}, \Delta T=1\right)$ states are not strongly excited in 'Tight nuclei. In the $\left(\vec{\gamma}, \gamma^{\prime}\right)$ experiment (3), in the energy range from 7.5 to $11.8 \mathrm{MeV}$, there are at least nine $1^{-}$states, the strongest one being at $7.896 \mathrm{MeV}$. The predicted $\left(p, p^{\prime}\right)$ cross section for this state at $4^{\circ}$ is very small $\left(0.15 \mathrm{mb} . \mathrm{sr}^{-1}\right)$ and is in very good agreement with the experimental value of $0.15 \pm 0.02 \mathrm{mb} . \mathrm{sr}^{-1}$. For the other weaker 1- states, the predicted $\left(p, p^{\prime}\right)$ cross sections are below the detection limit and in fact are not observed in the present experiment. We conclude therefore that all the observed states that have a forward peaked angular distribution, are $1^{+}$states. In ${ }^{54} \mathrm{Fe}$, as in ${ }^{52} \mathrm{Cr}$, the strongest $1^{-}$state reported in the $\left(\vec{\gamma}, \gamma^{\prime}\right)$ experiment, has a $\left(p, p^{\prime}\right)$ cross section at the limit of detectability. This provides further confirmation that all the states observed are indeed $1^{+}$states. For the relative strengths of the $1^{+}$states, the agreement with the electromagnetic measurements is generaliy good for the three nuclei. The comparison for $50 \mathrm{Ti}$ is illustrated in Fig. 9 where the $\left(p, p^{\prime}\right)$ differential cross sections at $4^{\circ}$ are compared wi th the $B(M 1)$ values extracted from inelastic electron scattering (30). For the states described by neutron spin-flip configurations, the agreement is quite good. For the proton spin-flip states, due to the orbital term involved in the electromagnetic M1 operator, there are strong differences. A complete report on these nuclei will be pubiished shortly (13). Another interesting case is ${ }^{51} \mathrm{~V}$ which has three protons on the $f_{7}$ shel]. In ( $\left.p, p^{\prime}\right)$ scattering at $201 \mathrm{MeV}(8)$, a strong bump with some fine structures is observed centered at $10.15 \mathrm{MeV}$ excitation energy with a width at half maximum of about $1.35 \mathrm{MeV}$ (Fig. 10 The analogue of this resonance has been observed in a $(p, n)$ reaction (11). The $\left(e, e^{\prime}\right)$ spectrum (31) is shown for comparison in Fig. 10. In spite of a close search, no $M 1$ strength at all is observed in the $\left(e, e^{\prime}\right)$ experiment! This complete discrepancy for ${ }^{5} \mathrm{Y} V$ contrast sharply with the qualitative agreement obtained for the neighbouring even-even $(N=28)$ nuclei. It has been suggested (31) that in the (e, $\left.e^{\prime}\right)$ reaction, the destructive interference between the orbital and the spin term is in part responsible for the non observation of the M1 resonance. The MI resonance can also be simply missed below the limit of detection because of a poor peak to continuum ratio. 


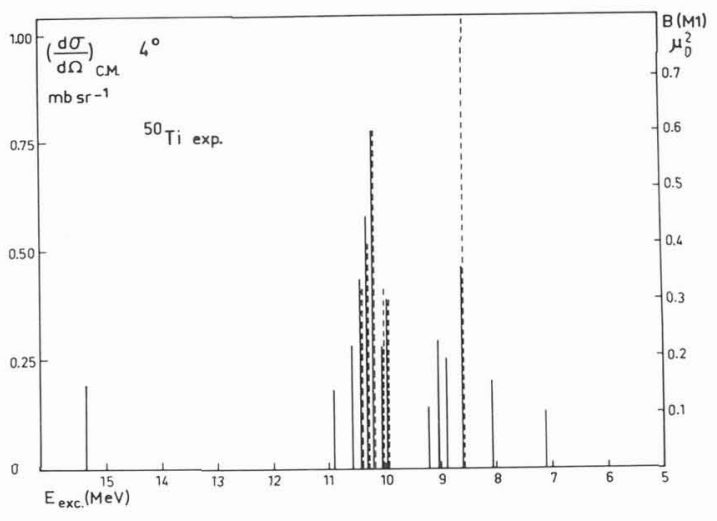

Fig. 9 : M1 strength distribution in ${ }^{50} \mathrm{Ti}$. The present $\left(p, p^{\prime}\right)$ cross sections are in full line, the $B(M 1)$ values obtained in the $\left(e, e^{\prime}\right)$ experiment (30) are in dashed line.

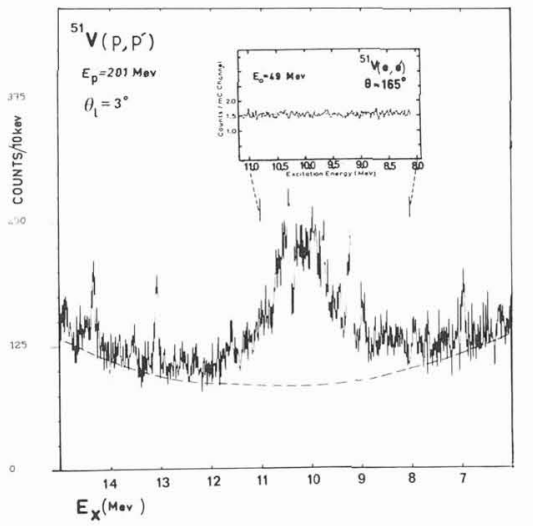

Fig. 10 : Discrepancy between $\left(e, e^{\prime}\right)$ (31) and $\left(p, p^{\prime}\right)(8)$ concerning the $M 1$ Resonance in ${ }^{5} \mathrm{~V}$.

3) ${ }^{90} \mathrm{Zr}$ and ${ }^{88} \mathrm{Sr}$
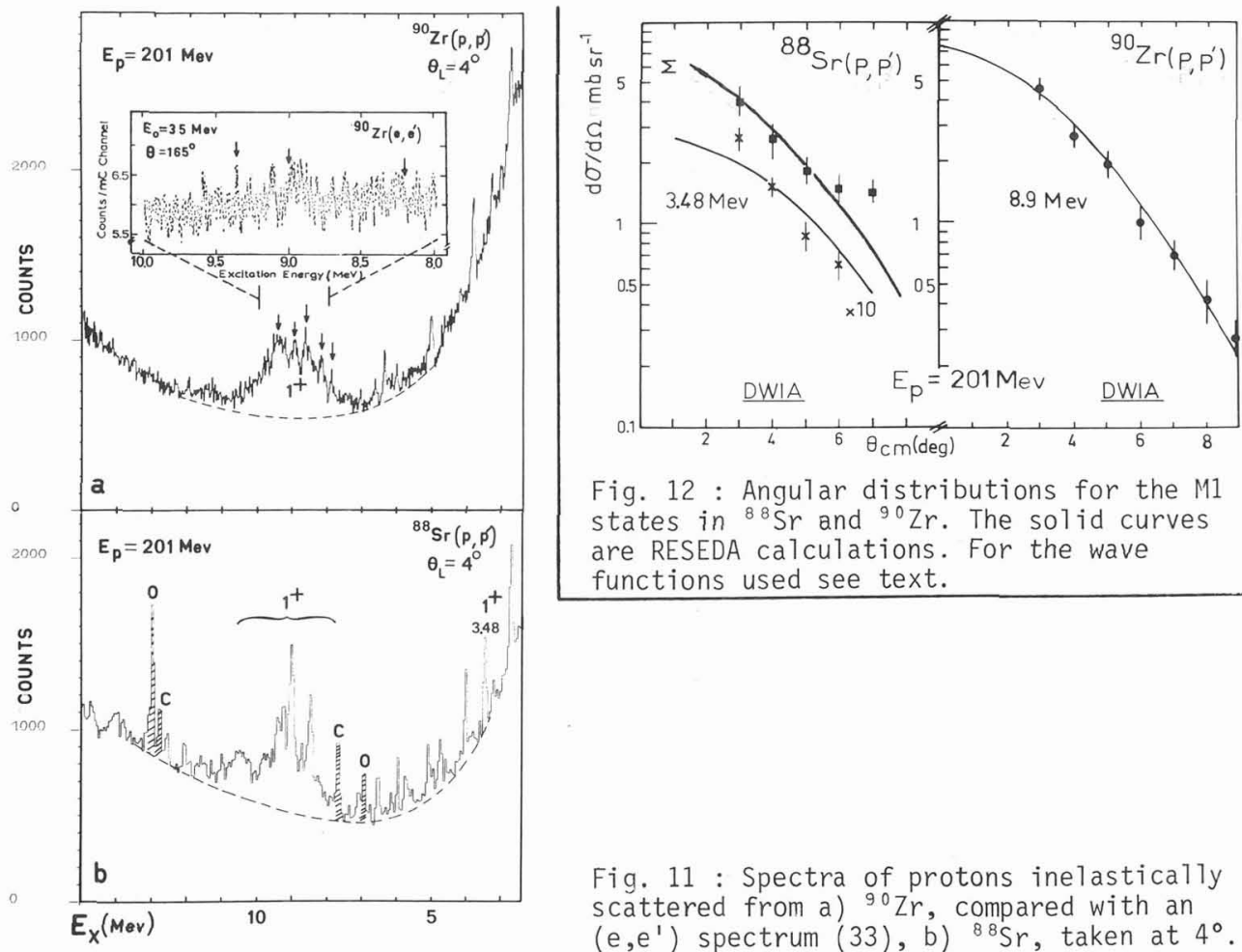

Fig. 12 : Angular distributions for the $M 1$ states in ${ }^{88} \mathrm{Sr}$ and ${ }^{90} \mathrm{Zr}$. The solid curves are RESEDA calculations. For the wave functions used see text.

Fig. 11 : Spectra of protons inelastically scattered from a) ${ }^{9}{ }^{\circ} \mathrm{Zr}$, compared with an $\left(e, e^{\prime}\right)$ spectrum $(33)$, b) ${ }^{8} 8 \mathrm{~S} r$, taken at $4^{\circ}$. 
The first medium heavy nucleus for which an M1 resonance was observed in ( $\left.p, p^{\prime}\right)(5)$ scattering was ${ }^{90} \mathrm{Zr}$. A large bump was located at an excitation energy of $8.9 \pm 0.2$ $\mathrm{MeV}$ with a width at half maximum of $1.5 \pm 0.2 \mathrm{MeV}$. The excitation energy of the MI resonance in ${ }^{0} \mathrm{Zr}$ agrees with the expected value both from the theoretical prediction of Bertsch (32) and from the position of the analogue state observed in the $(p, n)$ reaction. The $\left(e, e^{\prime}\right)$ experiments $(33)$ do not agree with the observation of a broad resonance, they instead identify only three definite $1^{+}$states respectively at $8.23,9.00$ and $9.37 \mathrm{MeV}$ and seven other possible $\left(1^{+}\right.$or $\left.2^{-}\right)$states. In a recent $\left(p, p^{\prime}\right)$ experiment at LAMPF (34) the resonance has been analysed into several individual peaks showing that in agreement with $\left(e, e^{\prime}\right)$, the $9.0 \mathrm{MeV}$ excitation energy region shows considerable fine structure but consistent disagreements still remain. In the present $\left(p, p^{\prime}\right)$ experiment, done with very good statistics and an energy resolution of $60 \mathrm{keV}$, fine structures are observed on the bump, they are denoted by arrows in the spectrum given in Fig. 11. Some of the structures correspond to the reported ( $e, e^{\prime}$ ) transitions, however in spite of the good energy resolution, no definite peak can be clearly isolated in the bump. Once again, concerning a broad resonance, we have strong disagreements with $\left(e, e^{\prime}\right)$ results.

The measured angular distribution for the M1 resonance in ${ }^{9}{ }^{\circ} \mathrm{Zr}$ is shown in Fig. 12 and is well fitted by a DWIA calculation using a simple $v\left(g_{7 / 2}, g_{9 / 2}^{-1}\right)$ configuration. The normalization factor is 0.3 . Ponomarev et a 1 . (35) have pointed out that the admixture of proton configurations can increase the normalisation factor.

${ }^{8} \mathrm{Sr}$ is an especially inferesting nucleus because, first a low energy $1^{+}$state, due mainly to a $\pi\left(2 p_{1 / 2}, 2 p_{3 / 2}^{-1}\right)$ configuration, is expected (this state has been observed in (e, $\mathrm{e}^{\prime}$ ) scattering (36)), secondly as ${ }^{88} \mathrm{Sr}$ has the same number of neutrons as ${ }^{90} \mathrm{Zr}$, a similar MI resonance is also expected. The ${ }^{8} 8 \mathrm{Sr}\left(\mathrm{p}, \mathrm{p}^{\prime}\right)$ spectrum is shown in Fig. 11 ; one can observe the low energy Ml transition at $3.48 \mathrm{MeV}$ and at higher energy, a broad resonance centered at $8.90 \pm 0.15 \mathrm{MeV}$. Compared to $90 \mathrm{Zr}$, many fine structures clearly appear on the resonance, respectively at $8.65,9.05,9.30$ and $9.47 \mathrm{MeV}$ excitation energy. Electron scattering measurements in this excitation energy region would be of great interest for comparison. The summed cross section $(\Sigma)$ for the resonance and the superposed structures has, with in the experimental uncertainties, the same value as the resonance in ${ }^{90} \mathrm{Zr}$. Assuming a simple $v\left(g_{7 / 2}, g_{9 / 2}^{-1}\right)$ configuration, the angular distribution has been fitted by a DWIA calculation (Fig.12). About $30 \%$ of the predicted strength is observed. For the $1^{+}$state at $3.48 \mathrm{MeV}$, a DWIA calculation, using a BROKEN PAIR MODEL wave function (36) is given in Fig.12. Further analysis are actually under progress.

\section{4) The controversial case of ${ }^{208} \mathrm{~Pb}$}

The missing M1 strength in $208 \mathrm{~Pb}$ has been a controversial and a long standing problem. In a recent $\left(\vec{\gamma}, \gamma^{\prime}\right)$ experiment $(37)$, the existence of a low lying $1^{+}$state at $5.846 \mathrm{MeV}$ has been established. This $1^{+}$state has also been observed in low energy $\left(p, p^{\prime}\right)(38)$ and $\left(e, e^{\prime}\right)$ (39) reactions. In Fig. 13, is given a spectrum obtained in a very recent $\left(\mathrm{p}, \mathrm{p}^{\prime}\right)$ measurement done at $201 \mathrm{MeV}$. Contrarily to the other cases presented, the $208 \mathrm{~Pb}$ spectrum at forward angles is mainly dominated by strong $\left(1^{-}, \Delta T=1\right)$ states excited through the CouTomb interaction. At high excitation energy, we observe the large Giant Dipole Resonance and at low excitation energy, the strongest $1^{-}$states already reported in the $\left(\vec{\gamma}, \gamma^{\prime}\right)$ experiment.

In Fig. 14, we see how accurately the predicted ( $\left.p, p^{\prime}\right)$ angular distribution fits the measured one for the strong $1^{-}$state at $5.514 \mathrm{MeV}$. Concerning the $1^{+}$state at $5.846 \mathrm{MeV}$, there are two possible wave functions which lead to either an "isoscalar" or an "isovector" nature for this state (39). We have analysed the present $\left(p, p^{\prime}\right)$ results with the two configurations. We see in $\mathrm{Fig.} 14$ that both calculations reproduce the shape of the measured anguiar distribution, however the isovector wave function underestimates the observed cross section by almost a factor of two $(N=2.0)$. Because of this unusual value of $N$ (generally $N=0.3$ to 0.5 ) we exclude the possibjlity of an isovector nature for this state. The isoscalar calculation has to be 


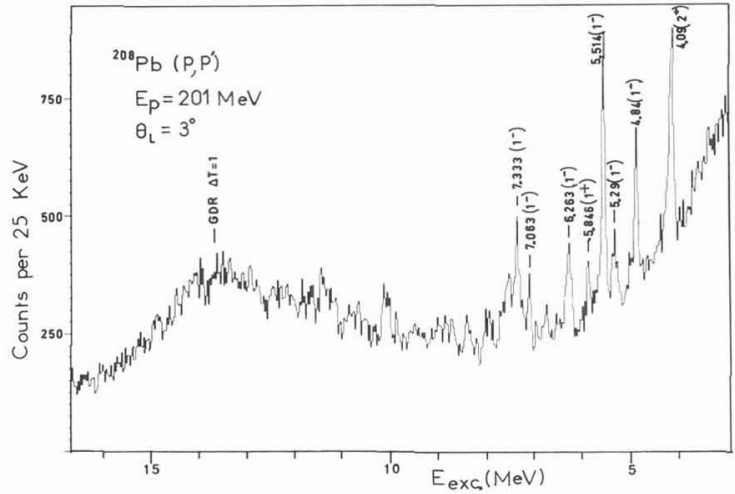

Fig. $13:{ }^{208} \mathrm{~Pb}\left(\mathrm{p}, \mathrm{p}^{\prime}\right)$ spectra taken at $3^{\circ}$. The energies of the excited states are taken from the $\left(\vec{\gamma}, \gamma^{\prime}\right)$ experiment (37).

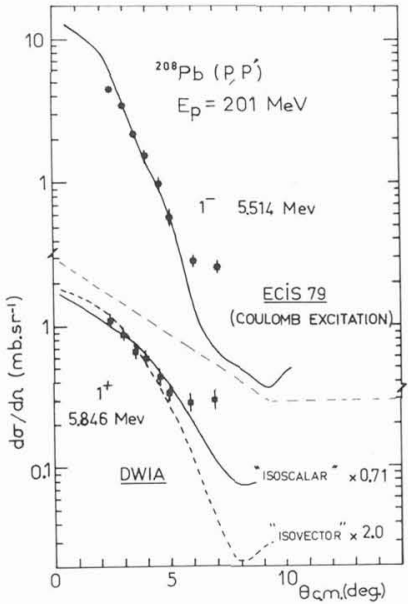

Fig. 14 : Angular distribution for a $1^{-}$and the $1^{+}$state in $208 \mathrm{pb}$. For the theoretical calculations see text.

normalized by 0.71 . Better descriptions for this state are needed in order to be sure about the missing strength in the isoscalar channel. A further argument in favour of the isoscalar nature of this state is given in a $\left(d, d^{\prime}\right)$ experiment, where this state is observed (38 b). The present ( $\left.p, p^{\prime}\right)$ results are preliminary and detailed analys is of the excitation energy region from 7 to $10 \mathrm{MeV}$ is under progress.

\section{IV - SUMMARY AND CONCLUSION}

Intermediate energy $\left(p, p^{\prime}\right)$ scattering at forward angles appears to be highly selective for exciting spin-flip transitions. The strong enhancement of the observed structures or resonances for bombarding energies near $200 \mathrm{MeV}$ is consistent with M1 transitions mediated essentially through the $V_{\text {a }}$ part of the nucleon-nucleon interaction. All the observed transitions have a forward peaked angular distribution, characteristic of a $\Delta \mathrm{L}=0$ transfer. In 1 ight nuclei by comparison with $\left(\vec{\gamma}, \gamma^{\prime}\right)$ results, it is shown that no strong $\left(1^{-}, \Delta T=1\right)$ state is excited. The situation is however different in heavy nuclei where El transitions excited through the Coulomb interaction, are dominant. In nuclei where the M1 transitions appear as fine structures or peaks in the $\left(p, p^{\prime}\right)$ experiment, there is an overall agreement with $\left(e, e^{\prime}\right)$ results. In opposition, when broad MI resonances are reported in $\left(p, p^{\prime}\right)$, no such resonances are seen in $\left(e, e^{\prime}\right)$; the clearest case being that of ${ }^{5} \mathrm{l}$. Although in $\left(p, p^{\prime}\right)$ the predicted strength is strongly model dependent and no theoretical calculation for the continuum is actually available, ${ }^{48} \mathrm{Ca}$ appears as the ideal case to study the missing strength, at most $40 \%$ of the predicted strength is observed in this nucleus. In almost al7 the other nuclei studied, only 25 to $30 \%$ of the expected strength is observed. This is smaller than the $50 \%$ reported in the $(p, n)$ reactions, however we must keep in mind that $\left(p, p^{\prime}\right)$ reactions are much more sensitive to the correlations in the ground state. In ${ }^{20}{ }^{8} \mathrm{~Pb}$, the $1^{+}$state at $5.846 \mathrm{MeV}$ is observed and detailed analysis of the higher excitation energy region is presently in progress.

\section{$V$ - ACKNOWLEDGEMENTS}

The results discussed in this paper are a result of the collaboration of many people. The ${ }^{8} \mathrm{Sr}\left(p, p^{\prime}\right)$ experiment was done in an ORSAY-VRIJE Universiteit collaboration. 
All the other experiments were done in an ORSAY-MSU collaboration. I shall here specifically give the names of people involved in these experiments. N. Marty, M. Morlet, A. Willis and J.C. Jourdain (ORSAY); N. Anantaraman, G.M. Crawley, A. Galonsky and J.E. Duffy (MSU); H.P. Blok and L.R. Kouw (Vrije Universiteit).

This work was partly supported by the U.S-N.S.F. under grants PHY-80-17605, INT-8116064 and INT-82-63242.

\section{REFERENCES}

1) G. Eulenberg et a]., Phys. Lett. 116 B (1982) 113.

A. Richter, Workshop on giant resonances, Mainz, June 1982.

A. Richter, Nucl. Phys. A374 (1982) $177 \mathrm{C}$.

2) C. Gaarde et a1., Nucl. Phys. A369 (1981) 258.

C.D. Goodman and S.D. Bloom, Int. Conf. on Spin excitation, Telluride March 1982 and references therein.

3) U.E.P. Berg et al., Phys. Lett. 103B (1981) 301.

U.E.P. Berg et al., Nucl. Phys. A398 (1983) 397.

U.E.P. Berg, Workshop on Giant Resonances, Mainz June 1982 and references therein.

4) G.P.A. Berg, Int. Conf. on Spin excitations, Telluride, March 1982

M. Fujiwara, Int. Conf. on Spin excitations, Telluride, March 1982.

5) G.M. Crawley et al., Phys. Rev. C26 (1982) 87.

N. Anantaraman et a1., Phys. Rev. Lett. 46 (1981) 1318.

N. Marty et a7., Nuct. Phys. A396 (1983) $145 \mathrm{C}$.

6) M. Ericson et al., Phys. Lett. 45B (1973) 19.

E. Oset and M. Rho, Phys. Rev. Lett. 42 (1979) 47.

A. Bohr and B. Mottelson, Phys. Lett. 100B (1981) 10.

A. Harting et a1., Phys. Lett. 104B (1981) 261.

7) K. Shimizu et aT., Nucl. Phys. A226 (1974) 282.

I.S. Towner and F.C. Khama, NucT. Phys. A399 (1983) 334.

8) C. Djalali et al., Nucl. Phys. A388 (1982) 1.

9) R. Schaeffer and J. RaynaT, CEN, Saclay Report, unpublished.

10)W.G. Love and M.A. Franey, Phys. Rev. C24 (1981) 1073.

11) C. Gaarde, private communication.

12) S. Imanishi et al., RCNP, Annual Report (1981) 43.

13) C. Djalali et al., Nucl. Phys., to be published.

14) C. Djalali et al., Z. Phys. A298 (1980) 79.

15) J. Raynal, Phys. Rev. C23 (1981) 2571, and private communication.

16) F. Osterfeld, Int. Conf. on spin Excitations in Nuclei, Tel]urjde, March 1982.

17) C. Gaarde, Nucl. Phys. A396 (1983) 127 C.

18)W. Steffen et a1., Phys. Lett. 95B (1980) 23.

19) G.M. Crawley et al., Phys. Lett. 127B (1983) 322.

20) Y. Fujita et al., Phys. Rev. C25 (1982) 678. 
21) J.B. Mc. Grory and B.H. Wildentha1, Phys. Lett. 103B (1981) 173.

22) A. Willis, Thesis, Univ. of Paris, (1968) unpubitished.

23) M. Lacombe et al., Phys. Rev. C21 (1980) 861, and private communication.

24) W.G. Love, private communication.

25) G.P.A. Berg et ar., Phys. Rev. C25 (1982) 2100.

26) S. Brandenburg et a1., Int. Conf. HESANS, sept. 83, Abstract 3.3.

27) C.D. Goodman et a1., Phys. Lett. 107B (1981) 406.

28) N. Anantaraman et a1., Int. Conf. HESANS, sept. 83, Abstract 6.1.

29) B.C. Metsch and W. Knüpfer, private communication.

30) W. Steffan and A. Richter, private communication.

31) D. Benda et a1., Nucl. Phys. A398 (1983) 408.

32) G.F. Bertsch, Nucl. Phys. A354 (1981) 157.

33) D. Meuer et aT., Nucl. Phys. A349 (1980) 309.

S. Muiller et a1., Phys.. Lett. I13B (1982) 362.

34) S. Nanda et a1., Int. Conf. HESANS, sept. 83, Abstract 6.15.

35) V. Yu: Ponomarev et a1., Int. Conf. HESANS, sept. 83, Abstract 7.9.

36) L.T, van der Bijl et a1., Z. Phys. A305 (1982) 231.

37) K. Wienhard et a1., Phys. Rev. Lett. 49 (1982) 18.

38a)S. I. Hayakawa et a1., Phys. Rev. Lett. 49 (1982) 1624.

b)G.P.A. Berg et al., RCNP Int. Symp. on Light Ion Reaction Mechanism, Osaka, May 1983.

39) S. Müller et a1., Phys. Lett. 120B (1982) 305. 